Provided for non-commercial research and education use. Not for reproduction, distribution or commercial use.

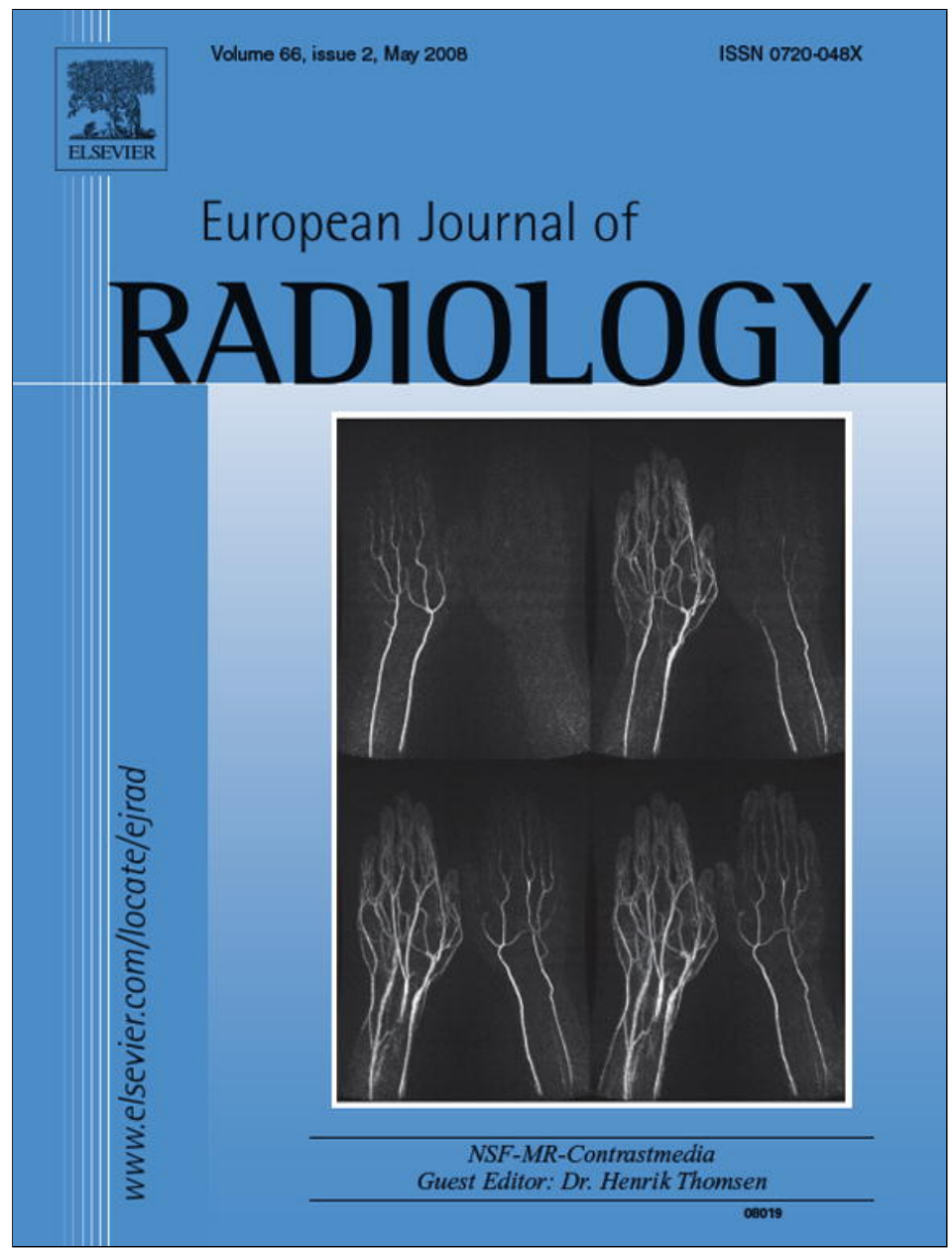

This article appeared in a journal published by Elsevier. The attached copy is furnished to the author for internal non-commercial research and education use, including for instruction at the authors institution and sharing with colleagues.

Other uses, including reproduction and distribution, or selling or licensing copies, or posting to personal, institutional or third party websites are prohibited.

In most cases authors are permitted to post their version of the article (e.g. in Word or Tex form) to their personal website or institutional repository. Authors requiring further information regarding Elsevier's archiving and manuscript policies are encouraged to visit:

http://www.elsevier.com/copyright 


\title{
Comparison of manual direct and automated indirect measurement of hippocampus using magnetic resonance imaging
}

\author{
Frederik L. Giesel ${ }^{\mathrm{a}, \mathrm{b}, *, 1}$, Philipp A. Thomann ${ }^{\mathrm{c}, 1}$, Horst K. Hahn ${ }^{\mathrm{d}}$, Maria Politi ${ }^{\mathrm{e}}$, \\ Bram Stieltjes $^{\mathrm{a}}$, Marc-André Weber ${ }^{\mathrm{a}}$, Johannes Pantel ${ }^{\mathrm{f}}$, I.D. Wilkinson ${ }^{\mathrm{b}}$, \\ Paul D. Griffiths ${ }^{b}$, Johannes Schröder ${ }^{c}$, Marco Essig ${ }^{a}$ \\ a Department of Radiology, German Cancer Research Center, Germany \\ ${ }^{\mathrm{b}}$ MRI Unit, Department of Radiology, Sheffield, UK \\ ${ }^{\mathrm{c}}$ Section of Geriatric Psychiatry, University of Heidelberg, Germany \\ d MeVis, Bremen, Germany \\ e Neuroradiology, Homburg/Saar, Germany \\ ${ }^{\mathrm{f}}$ Department of Psychiatry, University of Frankfurt, Germany
}

Received 28 February 2007; received in revised form 9 May 2007; accepted 8 June 2007

\begin{abstract}
Purpose: Objective quantification of brain structure can aid diagnosis and therapeutic monitoring in several neuropsychiatric disorders. In this study, we aimed to compare direct and indirect quantification approaches for hippocampal formation changes in patients with mild cognitive impairment and Alzheimer's disease (AD).

Methods and materials: Twenty-one healthy volunteers (mean age: 66.2), 21 patients with mild cognitive impairment (mean age: 66.6), and 10 patients with AD (mean age: 65.1) were enrolled. All subjects underwent extensive neuropsychological testing and were imaged at $1.5 \mathrm{~T}$ (Vision, Siemens, Germany; T1w coronal TR $=4 \mathrm{~ms}$, Flip $=13^{\circ}$, FOV $=250 \mathrm{~mm}$, Matrix $=256 \times 256,128$ contiguous slices, $1.8 \mathrm{~mm}$ ). Direct measurement of the hippocampal formation was performed on coronal slices using a standardized protocol, while indirect temporal horn volume (THV) was calculated using a watershed algorithm-based software package (MeVis, Germany). Manual tracing took about 30 min, semi-automated measurement less than 3 min time.

Results: Successful direct and indirect quantification was performed in all subjects. A significant volume difference was found between controls and $\mathrm{AD}$ patients $(p<0.001)$ with both the manual and the semi-automated approach. Group analysis showed a slight but not significant decrease of hippocampal volume and increase in temporal horn volume (THV) for subjects with mild cognitive impairment compared to volunteers $(p<0.07)$. A significant correlation $(p<0.001)$ of direct and indirect measurement was found.

Conclusion: The presented indirect approach for hippocampus volumetry is equivalent to the direct approach and offers the advantages of observer independency, time reduction and thus usefulness for clinical routine.
\end{abstract}

(C) 2007 Published by Elsevier Ireland Ltd.

Keywords: Magnetic resonance imaging; Computer assisted image analysis; Alzheimer's disease; Hippocampal measurement; Mild cognitive impairment

Abbreviations: MRI, magnetic resonance imaging; AD, Alzheimer's disease; THV, temporal horn volume; THI, temporal horn index

* Corresponding author at: German Cancer Research Center (DKFZ), Department of Radiology, INF 280, 69120 Heidelberg, Germany.

Tel.: +496221 422492; fax: +496221422462.

E-mail address: f.giesel@dkfz.de (F.L. Giesel).

1 These authors contributed equally.

\section{Introduction}

Alzheimer's disease (AD), the most frequent dementia beyond the age of 65 years, affects an eminent and increasing part of the population. With the availability of modern drug treatment, e.g. cholinesterase-inhibitors, and promising therapeutic options in future (e.g. anti-amyloid strategies), the development of sensitive and specific markers of early AD has gained importance. It is generally accepted that most of the subjects who develop $\mathrm{AD}$ pass through a transitional phase between health 
and clinically manifest dementia commonly referred as mild cognitive impairment.

Neuropathological research has shown that brain degeneration occurs very early in the course of the disease, even before the onset of clinical symptoms, and predominantly affects certain areas, particularly the substructures of the medial temporal lobe [1-3]. Previous neuroimaging studies reported significant atrophy of the hippocampus [4-7], the parahippocampal gyrus [8] and the entorhinal cortex [6,7,9-12] in subjects with mild cognitive impairment and AD. In the mentioned studies, image analysis was performed using the region of interest (ROI) approach which is based upon the a priori definition of specific brain regions. ROI analysis constitutes, up to now, the gold standard in brain atrophy measurements, though it necessitates highly developed neuroanatomical knowledge and is very labour intensive. Therefore it is difficult to apply in a clinical setting. Newer timesaving, almost fully automatic and therefore observer independent imaging techniques like voxel-based morphometry (VBM) allow for detection of volume or shape changes throughout the entire brain. However, having a $t$-test as the fundamental statistical tool, the latter method is unsuitable for the comparison of one subject with a normative template and thus solely allows group comparisons. The use of magnetic resonance imaging (MRI) in daily routine has so far been limited to exclude other pathologies that may cause dementia, such as normotensive hydrocephalus, tumor, vascular lesions or infectious diseases [13,14].

Until now, no clinically applicable, fast and reliable technique for the direct quantification of the hippocampal volume is available. The development of such a method is challenging particularly with regard to the separation of certain parts of the hippocampus from adjacent brain structures on MR images (e.g. separation of the hippocampal head from the amygdala and hippocampal tail to posterior parts of the thalamus).

Since the temporal horns of the cerebral ventricles are adjacent to the hippocampal formation, atrophy of the latter should lead to an increasing volume of the temporal horns. The purpose of the current study was to determine whether an easily applicable method of quantifying the temporal horn volume (THV) $[15,16]$ may be equivalent in detecting structural alterations of the hippocampus when compared to the conventional direct ROI approach.

\section{Methods}

\subsection{Subject selection}

Twenty-one subjects with mild cognitive impairment (mean age: 65.2, S.D. 0.9) and 21 cognitively intact control subjects (mean age: 65.3, S.D. 0.8) were recruited among the Heidelberg subsample of the interdisciplinary longitudinal study on adult development (ILSE) which is conducted in Heidelberg and Leipzig (Germany). Cognitive performance was assessed using a previously described extensive neuropsychological test battery [17] Mild cognitive impairment was defined by Levy's criteria of Aging-associated cognitive decline [18].
As an additional reference group 10 AD-patients (mean age: 63.8, S.D. 7.4) were drawn from a sample of patients consecutively admitted and investigated at the Section of Geriatric Psychiatry (University of Heidelberg). These patients were diagnosed as suffering from AD according to the NINCDSADRDA criteria [19] and were matched to the two other groups by age and gender. The clinical evaluation of all subjects included ascertainment of personal and family history as well as a thorough psychiatric, physical, neurological and neuropsychological examination. Individuals presenting with psychopathological symptoms other than cognitive deficits or taking psychotropic medication other than nootropics on a regular basis were excluded. History of ischemic heart disease, cancer and cerebrovascular risk factors (hypertension and diabetes) were carefully evaluated. All subjects were dominantly right handed .Evaluation of all patient data was performed under a research protocol reviewed and approved by the IRB of the University of Heidelberg.

\subsection{Magnetic resonance imaging}

All examinations were performed on a $1.5 \mathrm{~T}$ clinical MR scanner (Magnetom Vision, Siemens Medical Solutions, Erlangen). A T1-weighted coronal high-resolution volume acquisition was performed $\left(\mathrm{TR}=4 \mathrm{~ms}\right.$, Flip $=13^{\circ}$, $\mathrm{FOV}=250 \mathrm{~mm}$, Matrix $=256 \times 256,128$ contiguous slices, $1.8 \mathrm{~mm}$ ) and supplemented with axial T2-axial weighted and FLAIR-images.

\subsection{Manual tracing of the hippocampus}

Measures of the hippocampal formation were taken from a previous study on changes of the medial temporal lobe in mild cognitive impairment conducted by our group [8]. In this study, manual tracing was performed for each subject on 30-35 consecutive T1-weighted sections using the manual segmentation function of NMRWin software [20,21]. Hippocampal measures were initiated three slices rostrally to the point of fusion of the pedunculi cerebri and the pons. Caudally, outlining of the hippocampal formation was terminated at the level where the fornix and the hippocampus fuse. Thus, small proportions of the anterior (head) and posterior (tail) parts of the hippocampal formation were not sampled. Using the above described rostral and caudal boundaries however, facilitated a standardised sampling of the hippocampal formation on those slices, bearing the hippocampus clearly demarcated from adjacent brain structures. To address inter-individual differences in head size, the manually traced hippocampal volumes were corrected by dividing them by each subject's intracranial volume. The latter was assessed semi-automatically as has been described previously [20,21].

\subsection{Indirect measurement of the temporal horn volume using semi-automated IWT}

The T1-weighted volumetric datasets were analyzed with a combination of fast 3D marker based segmentation and histogram analysis. All of the image analysis methods have been 

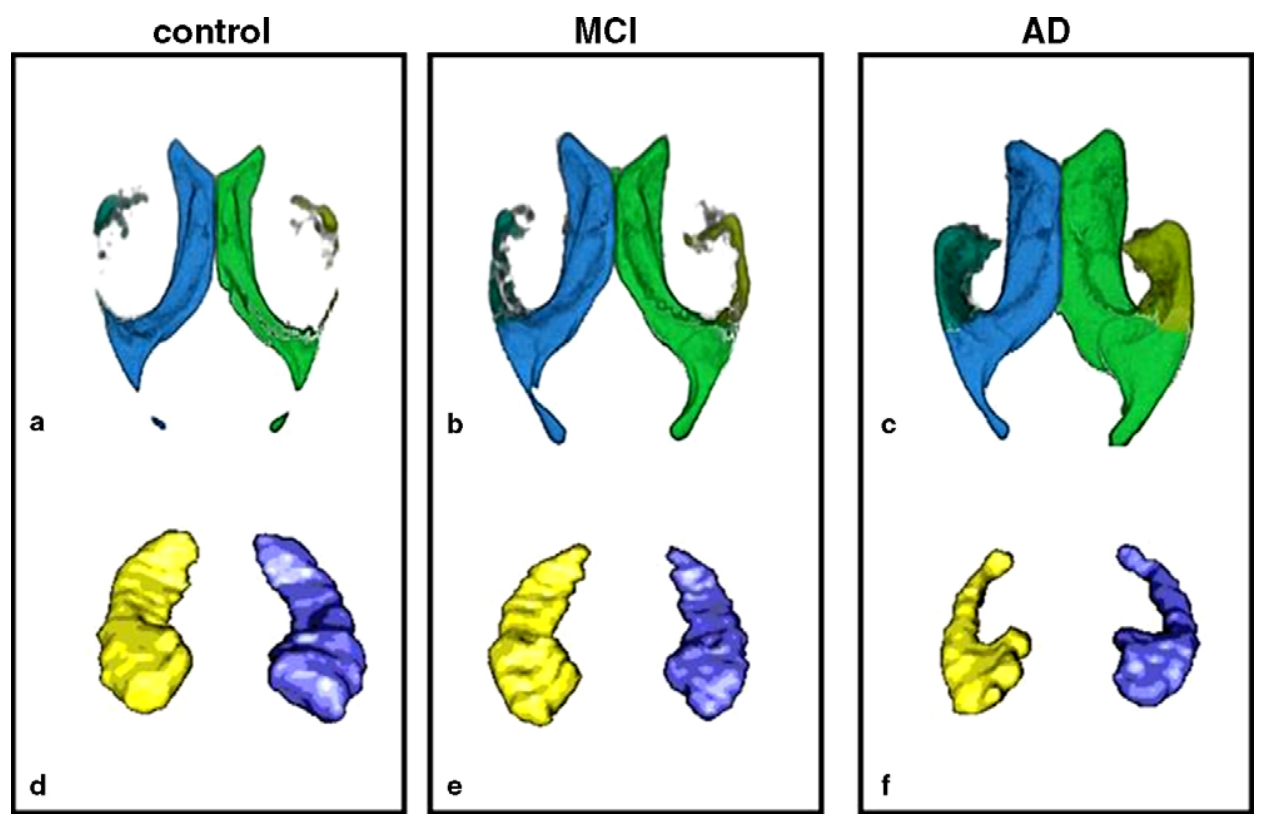

Fig. 1. Visualization of the direct $(\mathrm{a}-\mathrm{c})$ and indirect $(\mathrm{d}-\mathrm{f})$ measurement. Upper row: temporal horns in healthy control, mild cognitive impairment and AD. Lower row: corresponding hippocampal formations.

integrated as modules into the research and development platform MeVisLab (MeVis, Bremen, Germany) dedicated to rapid application prototyping in medical image analysis and visualization [16]. Segmentation of lateral ventricles including frontal horn, trigone, posterior horn, and temporal horn (TH) was performed applying an Interactive Watershed Transform (IWT) [15] to the original images. This requires two to six anatomical landmarks, depending on the image quality and width of the temporal horns, to segment the ventricles and was performed twice for every dataset, once with and once without the temporal horns, resulting in two volume estimates for each side, which were denominated LVV (lateral ventricular volume) and $\mathrm{LVV}^{*}$, respectively. The posterior TH limit was defined by a plane through three distinct anatomical landmarks, which are interactively identified on three synchronized orthogonal viewers. The landmarks are the anterior borders of the left and right trigone between frontal and temporal horn, and the posterior tip of the inferior colliculus. A fully automated analysis of regional histograms was applied to both segmentations (each containing two objects, left and right lateral ventricle) in order to robustly estimate $\mathrm{LVV}(\mathrm{L} / \mathrm{R})$ and $\mathrm{LVV}^{*}(\mathrm{~L} / \mathrm{R})$ [16]. The analysis was based on a trimodal Gaussian model (cerebro-spinal fluid, white, and gray matter) that explicitly includes partial volume terms, so-called Mixed Gaussians. Assuming uniformly distributed partial volume effects Mixed Gaussians take the form of plateau curves. The model is fitted to the histogram data by minimizing squared errors. As the raw hippocampal measures, THVs were corrected by the corresponding intracranial volume.

\subsection{Statistics}

Potential age differences between groups using MannWhitney $U$-test were explored. The right and left LVV, THV and THI of AD patients, subjects with mild cognitive impairment and controls were compared using Mann-Whitney $U$-test and Pearson's correlation coefficients were calculated between the direct and indirect morphometric measures. Statistical analysis was performed using SPSS version 11 (Chicago, IL). For Mann-Whitney $U$-test, a level of significance of $p<0.05$ was chosen.

\section{Results}

In all 52 cases, manual tracing of the hippocampal formation was performed and the ventricles were successfully quantified. A visualization of representative subjects' THV and its corresponding hippocampal formation is shown in Fig. 1; for illustrational purposes, in those subjects the hippocampi were completely traced. As elucidated in the methods section, demarcation of the head and the tail of the hippocampus to adjacent brain structures is often very difficult; therefore, we selected scans were the delineation was best possible. Manual tracing required 20-30 min, the semi-automated indi-

Table 1

Results of the direct and indirect methods of the MCI, AD and age-matched controls

\begin{tabular}{llll}
\hline Variable & Controls & MCI & AD \\
\hline THV right & $0.30(0.24)$ & $0.34(0.25)$ & $1.16(0.67)$ \\
THV left & $0.32(0.24)$ & $0.29(0.24)$ & $1.19(0.49)$ \\
THV mean & $0.62(0.46)$ & $0.63(0.46)$ & $2.35(0.96)$ \\
Hippocampus right & $2.18(0.37)$ & $2.08(0.42)$ & $1.39(0.27)$ \\
Hippocampus left & $2.13(0.25)$ & $2.16(0.40)$ & $1.23(0.20)$ \\
Hippocampus mean & $4.31(0.55)$ & $4.24(0.77)$ & $2.62(0.44)$ \\
\hline
\end{tabular}

THV: temporal horn volume; MCI: mild cognitive impairment; AD: Alzheimer's disease. Data reflect mean (S.D.). 


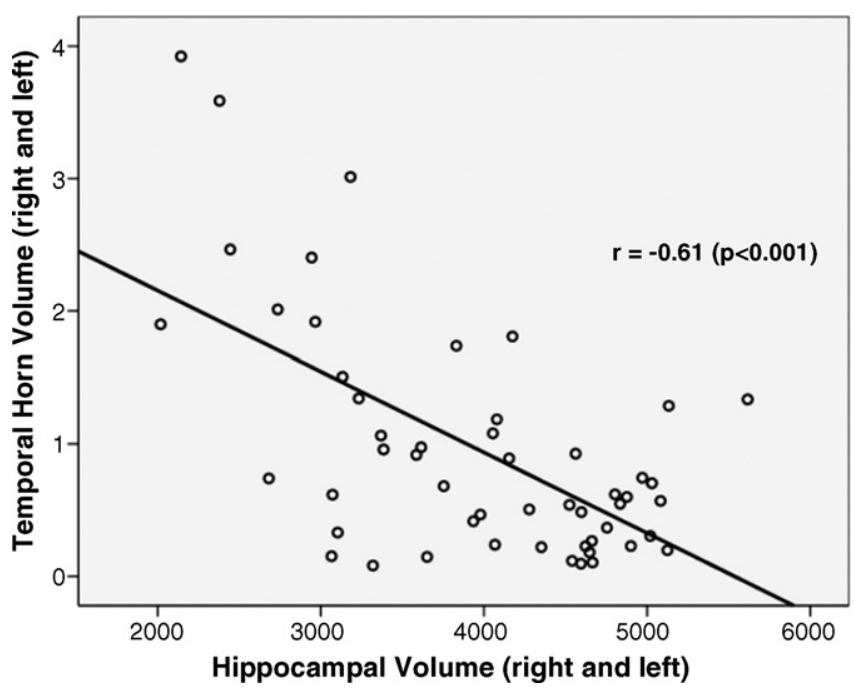

Fig. 2. Correlation of direct and indirect hippocampal volume $(p<0.001$; $\left.r=-0.588 ; r^{2}=0.345\right)$.

rect measurement took about 2-3 min for each MRI data set.

\subsection{Direct measurements}

A comparison of the volumetric measures of the three investigated groups is displayed in Table 1. According to the statistical analysis, there were no significant differences with respect to age, gender distribution, and level of school education. In addition, the total intracranial volume (TIV) did not differ significantly between the groups $(F=0.41 ;$ d.f. $=2,49 ; p=0.67)$. The (corrected) hippocampal volumes of the subjects with mild cognitive impairment were not significantly different when compared to the cognitively intact subjects. In contrast, the $\mathrm{AD}$ patients were characterized by significantly smaller hippocampal volumes bilaterally in comparison to both the mildly cognitive impaired and control group (hippocampus right: $F=13.5$, d.f. $=2,49 ; p<0.0005$; hippocampus left: $F=28.4$; d.f. $=2,49$; $p<0.0005)$.

\subsection{Indirect measurements}

The values for right and left LVV and THV of the controls, subjects with mild cognitive impairment and AD patients are summarized in Table 1. There was no significant difference between right, left and total LVV and THV of MCI subjects and controls $(p>0.05)$. The right, left and total LVV and THV of AD patients were significantly larger compared to controls $(p<0.05)$ and to subjects with MCI $(p<0.05)$.

\subsection{Correlation}

Direct (hippocampal volume) and indirect measurement (temporal horn volume) were significantly correlated $(p<0.001$; $r=-0.588 ; r^{2}=0.345$ ) (Fig. 2).

\section{Discussion}

In the present study, we ascertained structural alterations of the hippocampal formation on MR data sets using two different methodical approaches: (a) direct manual tracing on consecutive coronal slices and (b) indirect semiautomated estimation via computation of the temporal horn volume (THV). With regard to AD patients, direct measurement revealed a significant atrophy of the hippocampus when compared to healthy subjects. Accordingly, quantification of the temporal horn demonstrated a significantly increased volume in patients with AD. These findings are in line with the results of previous studies that investigated structural changes of the medial temporal lobe in $\mathrm{AD}$ [4,5,7-12].

In subjects with mild cognitive impairment, neither manual tracing nor measurement of the temporal horn revealed a significant difference when compared to healthy controls. This observation is contradictory to studies that reported significant loss of hippocampal volume already in mild cognitive impairment [4-7]. This divergent result may be related to aspects of sample selection: most importantly, our sample consisted of randomly selected subjects drawn from a population based sample, whereas the above mentioned studies predominantly analyzed MRI-scans of on demand patients who already might have been in a more advanced stage of their disorder.

As expected, comparison of the two different techniques revealed a significant negative correlation $(r=-0.588$, $p<0.001$ ), proving that enlargement of the lateral ventricles indirectly represents hippocampal atrophy. According to statistical analysis, approximately $65 \%$ of the variance remains unexplained. This is probably due to the fact that the temporal horns are not solely adjacent to the hippocampal formation but to further temporal regions, which underlie atrophic processes in the course of $\mathrm{AD}$, too.

Direct ROI analysis constitutes, up to now, the 'gold standard' in brain atrophy measurements, but there are some major disadvantages such as observer/operator dependency, expenditure of time and bias in anatomical region boundary selection [22]. So far, the variability of these measurements has limited direct comparison of the results of different research groups. Especially in case of the hippocampal formation direct manual tracing is a challenging task, as parts of its border are poorly differentiated on typical MR images [23].

As the substructures of the medial temporal lobe (hippocampus, parahippocampal gyrus, and entorhinal cortex) are known to be affected very early in the course of the disease, even years before the onset of clinical symptoms [1-3], the availability of an observer-independant method that facilitates the reliable assessment of structural alterations in this area would be very helpful for both research and clinical purposes.

In $\mathrm{AD}$, enlargement of the temporal horns of the lateral ventricles has been concordantly shown in various studies [24-28,13]. In three of the cited studies, measures of the hippocampal formation were performed, too $[27,29,30]$. Comparison of the accuracy in the differentiation of patients with $\mathrm{AD}$ from healthy controls yielded comparable or even superior results for temporal horn measures. In a follow-up study, Jack et al. [28] 
determined the annual rates of hippocampal and temporal horn volumetric changes in patients with $\mathrm{AD}$ compared to individually matched healthy controls. The rate of temporal horn enlargement was found to be nearly identical to that of hippocampal atrophy, indicating the temporal horn to be useful for longitudinal assessments.

In a recent study conducted by Thompson and colleagues [31], mapping of volumetric changes of the temporal horns served as a helpful measure of disease progression, linking with rates of cognitive decline in AD. Similar findings were reported by Jack et al. [32] who investigated 192 patients with AD in a 1 year MRI follow-up study; both studies found that change over time in MMSE performance linked even better with measures of THV than with changes of hippocampal measures. This might be explained by the fact that hippocampal atrophy rates diminish or even plateau in the course of $\mathrm{AD}$, while the temporal horns are still extending [31] due to ongoing atrophy in additional temporal regions.

In conclusion, these findings strongly support the usefulness of temporal horn measurements in both the diagnosis and monitoring of $\mathrm{AD}$.

Concerning their precision in correctly classifying patients and healthy controls, temporal horn maesures yielded varying results [24-26,29,30,32], which is probably related to aspects of sample selection (e.g. on demand patients versus population based study, different age cohorts), severity of the disease and image aquisition procedures, but also to different measurement protocols.

In the current study, we used a semi-automated technique for assessing the THV in patients with $\mathrm{AD}$, mild cognitive impairment and in healthy control subjects. The technique we used to evaluate the THV is simple, reliable, and independent of user experience. Moreover, our approach of temporal horn measurement is completed in 3 min and could therefore be applied in busy clinical settings. By contrast, manual segmentation of the hippocampal formation requires about 20-30 min, depending on the tracer's experience [33].

We previously showed that cerebral ventricular segmentation and volumetry based on the IWT and histogram analysis are largely independent of landmark positions [15]. Even though not fully automated, our method has shown an excellent intraand inter-observer as well as inter-examination reproducibility [15]. In this context it is important to note that even fully automated image analysis methods that are perfectly reproducible on a given data set by definition, may fail to provide reproducible measurements on repeated examinations. The only critical interactive steps comprised in our methodology are landmark definitions that an experienced user can perform rapidly and reliably, where, however, an automated method might fail in case of a noisy image or heavily deformed anatomy. Therefore, our method combines interactive with automated processing steps in a sensible fashion in order to maximize the precision and robustness of the derived results.

We propose our approach of THV estimation as an indirect, clinically easily applicable, sensitive and accurate regional measure for hippocampal atrophy with equivalent results as achieved by direct manual tracing.

\section{References}

[1] Braak H, Braak E. Neuropathological stageing of Alzheimer-related changes. Acta Neuropathol (Berl) 1991;82:239-59.

[2] Delacourte A, et al. The biochemical pathway of neurofibrillary degeneration in aging and Alzheimer's disease. Neurology 1999;52:1158-65.

[3] Van Hoesen GW, Augustinack JC, Dierking J, Redman SJ, Thangavel R. The parahippocampal gyrus in Alzheimer's disease. Clinical and preclinical neuroanatomical correlates. Ann NY Acad Sci 2000;911:254-74.

[4] Bottino CM, Castro CC, Gomes RL, Buchpiguel CA, Marchetti RL, Neto MR. Volumetric MRI measurements can differentiate Alzheimer's disease, mild cognitive impairment, and normal aging. Int Psychogeriatr 2002;14:59-72.

[5] De Santi S, et al. Hippocampal formation glucose metabolism and volume losses in MCI and AD. Neurobiol Aging 2001;22:529-39.

[6] Dickerson BC, Goncharova I, Sullivan MP, Forchetti C, Wilson RS, Bennett DA, Beckett LA, deToledo-Morrell L. MRI-derived entorhinal and hippocampal atrophy in incipient and very mild Alzheimer's disease. Neurobiol Aging 2001;22:747-54

[7] Du AT, et al. Magnetic resonance imaging of the entorhinal cortex and hippocampus in mild cognitive impairment and Alzheimer's disease. J Neurol Neurosurg Psychiat 2001;71:441-7.

[8] Pantel J, Kratz B, Essig M, Schroder J. Parahippocampal volume deficits in subjects with aging-associated cognitive decline. Am J Psychiat 2003;160:379-82.

[9] Toledo-Morrell L, Goncharova I, Dickerson B, Wilson RS, Bennett DA. From healthy aging to early Alzheimer's disease: in vivo detection of entorhinal cortex atrophy. Ann NY Acad Sci 2000;911:240-53.

[10] Juottonen K, Laakso MP, Insausti R, Lehtovirta M, Pitkanen A, Partanen $\mathrm{K}$, Soininen $\mathrm{H}$. Volumes of the entorhinal and perirhinal cortices in Alzheimer's disease. Neurobiol Aging 1998;19:15-22.

[11] Killiany RJ, Hyman BT, Gomez-Isla T, Moss MB, Kikinis R, Jolesz F, Tanzi R, Jones K, Albert MS. MRI measures of entorhinal cortex vs hippocampus in preclinical AD. Neurology 2002;58:1188-96.

[12] Xu Y, Jack Jr CR, O’Brien PC, Kokmen E, Smith GE, Ivnik RJ, Boeve BF, Tangalos RG, Petersen RC. Usefulness of MRI measures of entorhinal cortex versus hippocampus in AD. Neurology 2000;54:1760-7.

[13] Scheltens P. Early diagnosis of dementia: neuroimaging. J Neurol 1999;246:16-20.

[14] Frisoni GB. Structural imaging in the clinical diagnosis of Alzheimer's disease: problems and tools. J Neurol Neurosurg Psychiat 2001;70:711-8.

[15] Hahn HK, Peitgen HO. IWT - interactive watershed transform: a hierarchical method for efficient interactive and automated segmentation of multidimensional gray-scale images. Med Imag Image Process 2003;5032:643-53.

[16] Hahn HK, Rexilius J, Schlüter M. Fast and robust quantification of parahippocampal atrophy via temporal horn index. In: Proceedings of the BVM. Berlin: Springer; 2004. p. 371-5.

[17] Schroder J, Kratz B, Pantel J, Minnemann E, Lehr U, Sauer H. Prevalence of mild cognitive impairment in an elderly community sample. J Neural Transm Suppl 1998;54:51-9.

[18] Levy R. Aging-associated cognitive decline. Working party of the International Psychogeriatric Association in collaboration with the World Health Organization. Int Psychogeriatr 1994;6:63-8.

[19] McKhann G, Drachman D, Folstein M, Katzman R, Price D, Stadlan EM Clinical diagnosis of Alzheimer's disease: report of the NINCDS-ADRDA Work Group under the auspices of Department of Health and Human Services Task Force on Alzheimer's Disease. Neurology 1984;34:939-44.

[20] Friedlinger M, Schad LR, Bluml S, Tritsch B, Lorenz WJ. Rapid automatic brain volumetry on the basis of multispectral 3D MR imaging data on personal computers. Comput Med Imag Graph 1995;19:185-205.

[21] Friedlinger M, Schroder J, Schad LR. Ultra-fast automated brain volumetry based on bispectral MR imaging data. Comput Med Imag Graph 1999;23:331-7.

[22] Pruessner JC, Li LM, Serles W, Pruessner M, Collins DL, Kabani N, Lupien S, Evans AC. Volumetry of hippocampus and amygdala with highresolution MRI and three-dimensional analysis software: minimizing the discrepancies between laboratories. Cereb Cortex 2000;10:433-42. 
[23] Shen D, Moffat S, Resnick SM, Davatzikos C. Measuring size and shape of the hippocampus in MR images using a deformable shape model. Neuroimage 2002; 15:422-34.

[24] DeCarli C, Murphy DG, McIntosh AR, Teichberg D, Schapiro MB, Horwitz B. Discriminant analysis of MRI measures as a method to determine the presence of dementia of the Alzheimer type. Psychiat Res 1995;57:119-30.

[25] Erkinjuntti T, Lee DH, Gao F, Steenhuis R, Eliasziw M, Fry R, Merskey H, Hachinski VC. Temporal lobe atrophy on magnetic resonance imaging in the diagnosis of early Alzheimer's disease. Arch Neurol 1993;50:305-10.

[26] Frisoni GB, Beltramello A, Weiss C, Geroldi C, Bianchetti A, Trabucchi M. Linear measures of atrophy in mild Alzheimer disease. AJNR Am J Neuroradiol 1996;17:913-23.

[27] Frisoni GB, et al. Radial width of the temporal horn: a sensitive measure in Alzheimer disease. AJNR Am J Neuroradiol 2002;23:35-47.

[28] Jack Jr CR, Petersen RC, Xu Y, O'Brien PC, Smith GE, Ivnik RJ, Tangalos EG, Kokmen E. Rate of medial temporal lobe atrophy in typical aging and Alzheimer's disease. Neurology 1998;51:993-9.
[29] Scheltens P, et al. Atrophy of medial temporal lobes on MRI in "probable" Alzheimer's disease and normal ageing: diagnostic value and neuropsychological correlates. J Neurol Neurosurg Psychiat 1992;55:96772.

[30] Soininen H, Puranen M, Riekkinen PJ. Computed tomography findings in senile dementia and normal aging. J Neurol Neurosurg Psychiat 1982;45:50-4

[31] Thompson PM, et al. Mapping hippocampal and ventricular change in Alzheimer disease. Neuroimage 2004;22:1754-66.

[32] Jack Jr CR, et al. MRI as a biomarker of disease progression in a therapeutic trial of milameline for AD. Neurology 2003;60:25360

[33] Thacker NA, Varma AR, Bathgate D, Stivaros S, Snowden JS, Neary D, Jackson A. Dementing disorders: volumetric measurement of cerebrospinal fluid to distinguish normal from pathologic findings-feasibility study. Radiology 2002;224:278-85. 